\title{
Condición corporal y reinicio de la actividad ovárica posparto en vacas Holstein en Ecuador
}

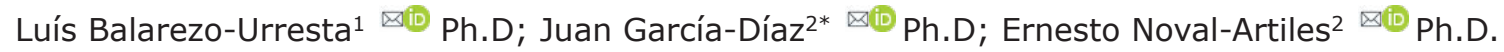

\begin{abstract}
1 Universidad Politécnica del Carchi. Facultad de Industrias Agropecuarias y Ciencias Ambientales. Tulcán, Proyecto de mejoramiento de la competitividad láctea en la provincia del Carchi. Escuela de Desarrollo Integral Agropecuario, Carchi, Ecuador.

2Universidad Central "Marta Abreu" de Las Villas. Facultad de Ciencias Agropecuarias, Departamento de Medicina veterinaria y Zootecnia, Carretera a Camajuaní Km. 5 1⁄2. Santa Clara. CP 54830, Santa Clara, Villa Clara, Cuba.

Correspondence: juanramon@uclv.edu.cu
\end{abstract}

Recibido: Noviembre 2019; Aceptado: Mayo 2020; Publicado: Agosto 2020.

\section{RESUMEN}

Objetivo. Determinar la influencia de la condición corporal al parto (CCP) en el reinicio de la actividad ovárica (RAO) posparto de la vaca Holstein en la región andina de Ecuador. Materiales y métodos. Se trabajaron 30 vacas. Se determinaron el momento de aparición del folículo dominante (FD), de ovulación y de actividad luteal; la duración del ciclo estral, el volumen del cuerpo lúteo (CL) y las concentraciones de progesterona $\left(\mathrm{P}_{4}\right)$ en suero sangíneo. Los estadígrafos descriptivos de las variables del RAO y sus indicadores se compararon según la CCP mediante una prueba de t-Student para muestras independientes. Se evaluó la asociación entre la CCP y el RAO posparto mediante un estudio caso control. Resultados. La duración del ciclo estral fue 23.10 días, el $46.67 \%$ de las vacas tuvo ciclos normales y el $53.33 \%$ ciclos anormales. El FD, la ovulación y la actividad luteal ocurrieron a los $16.63,27.76$ y 41.38 días posparto, respectivamente; antes $(p<0.05)$ en vacas con CCP $\geq 3.5$; en las que fueron mayores $(p<0.05)$ el volumen del $C L$ y las concentraciones de $P_{4}$. La CCP se correlacionó $(p<0.05)$ con los parámetros del RAO. Las vacas con CCP $<3.5$ puntos, tienen 10.50 veces más probabilidades de tener RAO tardío que las que tienen CCP $\geq$ de 3.5 puntos. Conclusiones. El RAO fue temprano, sobre el influyó la CCP, la que constituyó un factor de riesgo $(\mathrm{p}<0.05)$ para que las vacas tengan un RAO tardío.

Palabras clave: Folículo dominante; ovulación; cuerpo lúteo; progesterona; condición corporal; ciclo estral (Fuentes: DeCS, CAB).

\section{ABSTRACT}

Objective. To determine the influence of corporal composition at delivery (CCD) on the ovarian postpartum restart (OPR) of the Holstein cow in the Andes Region in Ecuador. Materials and methods. 30 cows were produced. All of the following moments were determined: the appearing of the dominant follicle (DF), the ovulation and luteal activity; the duration of the estrous cycle, the volume of the luteum body ( $L C)$ and the progesterone concentrations $\left(\mathrm{P}_{4}\right)$ on blood serum. Descriptive 
statisticians of the OPR variables and its indicators were compared according to the $\mathrm{BC}$, by means of a t-Student test for independent samples. The relationship between the BC and the postpartum OPR through a case-control case was assessed. Results. The duration of the oestrous cycle was 23.10 days, $46.67 \%$ of the cows had regular cycles and $53.33 \%$ were abnormal cycles. The DF, the ovulation and the luteal activity were seen at $16.63,27.76$ and 41.38 after postpartum, respectively; before $(p<0.05)$ on cows with $B C \geq 3.5$; in which both the $(p<0.05)$ and the volume of the LC and $P_{4}$ concentrations were higher. The $B C$ was correlated with $(p<0.05)$ with the OPR parameters. The cows with $<3.5 \mathrm{BC}$ points, are 10.50 times more prompt to have a late OPR than those with a $\geq$ de 3.5 points BC. Conclusions. The OPR was early, BC had an influence on it, which constituted a major $(p<0.05)$ risk factor on cows having a late OPR.

Keywords: Dominant follicle; ovulation; corpus luteum; progesterone; body condition; estrous cycle (Sources: DeCS, CAB).

\section{INTRODUCCIÓN}

Los eventos más notorios del reinicio de la actividad ovárica (RAO) son la presentación de celos, ovulación y desarrollo del cuerpo lúteo con su secresión de progesterona $\left(\mathrm{P}_{4}\right)(1)$. El RAO en la vaca la principal limitante en la eficiencia reproductiva y en el influyen varios factores, entre ellos, la nutrición y la condición corporal (CC) (2).

Existe escasa información sobre el RAO posparto en vacas en sistemas pastoriles; por esta razón, es necesario establecer los factores que influyen en el RAO en hembras bovinas en estas condiciones de producción para darles un manejo nutricional y reproductivo que permita alcanzar la máxima eficiencia reproductiva.

En los agroecosistemas pastoriles las interacciones de entre los componentes químicos en el suelo determinan el estado de los nutrientes en el pasto y en los animales que lo aprovechan. En estas condiciones las vacas lecheras tienen déficit energético y deficiencias o excesos de macro y microelementos que afectan su fertilidad (3).

La composición química del suelo y el pasto en agroecosistemas de la región andina de Ecuador originan en las vacas lecheras excesos de proteínas y deficiencias de energía, $\mathrm{P}, \mathrm{Cu}$ y $\mathrm{Zn}$; consecuentemente, estas presentan alteraciones nutricionales y metabólicas, y baja CC. En estos rebaños los principales indicadores reproductivos indican prolongación del anestro posparto $(4,5)$ además, la CC infliuye en la involución uterina (6), lo que indica que puede influir también sobre el RAO posparto.

El objetivo de este estudio fue determinar la influencia de la condición corporal al parto (CCP) en el RAO posparto de la vaca Holstein en la región andina de Ecuador.

\section{MATERIALES Y MÉTODOS}

Escenario de la investigación. El estudio se desarrolló de noviembre de 2015 y abril de 2016 en una hacienda de la parroquia Tufiño, cantón Tulcán, provincia El Carchi; ubicada entre los 10 '12' y $43^{\prime \prime}$ Latitud Norte (LN) y $78 \circ 33^{\prime} 12^{\prime \prime}$ Longitud Occidental (LW), a una altura de 2990 a $3450 \mathrm{msnm}$.

En el área experimental las precipitaciones anuales fluctúan entre 1000 y $1250 \mathrm{~mm}$ en el período lluvioso (PLL), de octubre a abril, y de 700 a $850 \mathrm{~mm}$ en el período poco lluvioso (PPLL), de mayo a septiembre. Las temperaturas mínimas y máximas son $2^{\circ} \mathrm{C}$ y $15^{\circ} \mathrm{C}$, respectivamente, las que oscilan entre 6 y $11^{\circ} \mathrm{C}(7)$.

La zona de estudio tiene suelo del orden Andisol con $70 \mathrm{~cm}$ profundidad efectiva. Su relieve es ondulado con porcentaje de pendiente entre 10 y $20 \%(4)$.

Sistema de alimentación y manejo. Los animales se mantuvieron en pastoreo racionado, con una carga global de 2.5 UGM $\mathrm{ha}^{-1}$. Predominaban los pastos naturalizados, Pennisetum clandestinum L. y Holcus lannatus L., y los mejorados Lolium perenne L. y sus cultivares "One fifty", Dactylus glomeratta L. y "Banquete", Trifolium repens L. y No se administró suplementación energético mineral.

Los pastos se regaban con agua de rio y se fertilizaban con urea en dosis de $200-300 \mathrm{~kg}$ de

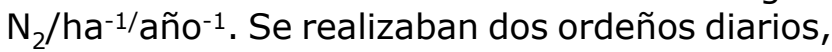
de forma manual, el primero de 4 a.m. a 7 a.m. y el segundo de 3 p.m. a 6 p.m. Los terneros permanecían con sus mdres hasta el tercer día de nacido, a partir del cual se se mantenían en crianza artificial. 
Animales utilizados. Se seleccionaron 30 vacas Holstein entre segunda y cuarta lactancia, con edad comprendida entre 4 y 8 años, nivel productivo de 15-18 L/vaca-1 día-1, CCP entre 3,0 y 4,0 en la escala de 5 puntos y clínicamente sanas. Se estableció el RAO posparto y su asociación con la CCP.

Para determinar el estado general de salud se consideraron todos los eventos vinculados con el parto y el puerperio, a los que se les dio seguimiento, aplicando las invariantes funcionales del método clínico, que incluyeron reseña, anamnesis, inspección y palpación; así como, percusión y aoscultación de órganos y sistemas. Además, se valorarron las desparasitaciones y vacunaciones según cronograma del área.

Toma de muestras de sangre y determinación de progesterona $\left(\mathrm{P}_{4}\right)$. La sangre se extrajo por venopunción coccígea con tubos vacutainer, previa limpieza y desinfección del área con alcohol al 70\%. Se utilizaron 10 - $50 \mu \mathrm{L}$ de suero sanguíneo y las concentraciones de $\mathrm{P}_{4}$ se determinaron cada siete días, desde el septimo hasta los 120 días posparto (DPP).

La $\mathrm{P}_{4}$ determinó por Electro quimioluminiscencia, en un analizador Cobas e 411 (Roche, Alemania), según los procedimientos del fabricante. Este método tiene elevada precisión ( $\mathrm{CV}<10 \%)$, y rango de detección amplio (0-6 ng/mL).

Condición Corporal. La CCP se estimó por inspección y palpación, clasificándola en la escala de 1-5 puntos y divisiones de 0.25 entre ellos según la metodología propuesta por Rodenburg (8).

Actividad ovárica. El RAO posparto se estableció cuando el diámetro de los folículos dominantes (FD) fue $\geq 5 \mathrm{~mm}$; éstos se estudiaron mediante ecografía hasta que se atresiaron u ovularon, o cuando las concentraciones de $\mathrm{P}_{4}$ fueron mayores que $1 \mathrm{ng} / \mathrm{mL}$ (9).

La primera ovulación se estableció cuando desapareció el folículo preovulatorio y en su lugar apareció un CL (10). También cuado las concentraciones de $\mathrm{P}_{4}$ en suero sanguíneo fueron mayores que $1 \mathrm{ng} / \mathrm{mL}$ (9).

El inicio de la actividad luteal se estableció cuando la concentración de $\mathrm{P}_{4}$ en suero sanguíneo fue mayor que a $1 \mathrm{ng} / \mathrm{mL}$ durantes dos muestreos consecutivos (11).
Los ciclos estrales después de la primera ovulación se consideraron normales cuando su duración fue entre 18 y 24 días o anormales (disciclia) cuando fueron $\leq$ a $170 \geq 25$ días (12).

Las vacas se clasificaron en dos categorías de acuerdo con el momento del RAO posparto, según las recomendaciones de Guáqueta et al (13), que para ello propone la $\bar{x} \pm D E$ de los días a la primera ovulación. Este evento ocurrió en las hembras en estudio a los $27.76 \pm 7.73$ días, por lo que se consieraron vacas con RAO temprano, las que ovularon dentro de los primeros 35 días y con RAO tardío las que lo hicieron posteriormente.

El diámetro del FD y el volumen del CL se calcularon con ecografía dos veces por semana; se utilizó un ecógrafo CTS-800 (SIUI, China), con un transductor lineal de $5 \mathrm{MHz}$, que brinda buena relación entre la calidad de la imagen y profundidad, hasta los $11 \mathrm{Cm}$ (14). El ecógrafo utilizado proporciona las medidas de estas estructuras ováricas automáticamente.

Procedimiento estadístico. Se calcularon la media $(\bar{x})$, desviación estándar (DE) y coeficiente de variación (CV) de cada variable del RAO. Se realizó una correlación simple entre la CCP y los principales indicadores de la RAO posparto y sus indicadores se compararon según la CCP con la prueba t-Student para muestras independientes. En estos procesamientos se utilizó el programa estadístico Statgraphics Centurion Ver. XV.II.

Se ejecutó un estudio caso control para determinar la asociación de la CCP de las vacas y el RAO posparto. Se obtuvieron las medidas de frecuencia, impacto y significación estadística con la utilización del paquete estadístico EPIDAT 3.1.

\section{RESULTADOS}

El primer FD apareció a los 16.63 días, con diámetro de $5.67 \pm 0.30 \mathrm{~mm}$, que alcanzó su mayor tamaño a los 11.26 días y la ovulación ocurrió 11 días después de aparecer el FD, a los 27.76 días. El ciclo estral sucesivo a la primera ovulación tuvo

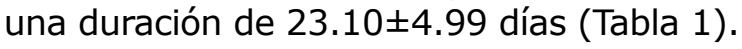

El $46.67 \%$ de las vacas tuvieron un ciclo estral que durò entre 18 y 24 días, mientras que el $53.33 \%$ restante presentó ciclos con duración menoro superior a este rango; de ellas, el $6.67 \%$ $\leq$ que 17 días, el $40 \%$ entre 25 y 30 días y $6.67 \%$ mayor de 30 días (Tabla 2). 
Tabla 1. Comportamiento de los principales indicadores del RAO posparto de vacas lecheras Holstein.

\begin{tabular}{lccc}
\hline \multicolumn{1}{c}{ Parámetros } & $\overline{\mathrm{x}}$ & DE & CV \\
\hline Aparición del FD (días) & 16.63 & 3.83 & 23.06 \\
Diámetro del FD en la emergencia $(\mathrm{mm})$ & 5.67 & 0.30 & 5.36 \\
Mayor diámetro del FD (mm) & 11.26 & 0.50 & 4.51 \\
Ocurrencia de la ovulación (días) & 27.76 & 7.73 & 28.58 \\
Actividad luteal (días) & 34.73 & 9.24 & 26.61 \\
Volumen del cuerpo lúteo (cm $\left.{ }^{3}\right)$ & 7.02 & 2.07 & 29.51 \\
Progesterona sérica (ng/mL) & 3.74 & 1.42 & 38.16 \\
Duración del ciclo estral (días) & 23.10 & 4.99 & 21.61 \\
\hline
\end{tabular}

Tabla 2. Distribución de frecuencias de la duración ciclo estral (días).

\begin{tabular}{ccccc}
\hline Intervalos & FR & FA & Clasificación & Meta (\%) \\
\hline$\leq 17$ & 0.0667 & 0.0667 & Cortos & $<10$ \\
$18-24$ & 0.4667 & 0.5333 & Normales & $65-70$ \\
$25-30$ & 0.4000 & 0.9333 & Largos & $<10$ \\
$>30$ & 0.0667 & 1.0000 & Largos & $<10$ \\
\hline
\end{tabular}

FR: Frecuencia relativa. FA: Frecuencia acumulada. La clasificación y la meta según los parámetros establecidos por Adams et al (12).

En las vacas con CCP $\geq 3.5$ apareció el FD a los 15.35 días y ocurrieron las primeras ovulaciones y la actividad luteal a los 23.94 y 29.64 días, por ese órden; todos estos eventos acontecieron más temprano $(\mathrm{p}<0.05)$ en las que tenían una CCP $<3.5$ (Tabla 3).

Tabla 3. Actividad ovárica posparto $(\overline{\mathrm{X}} \pm \mathrm{EE})$ de vacas lecheras Holstein según la condición Corporal al Parto.

\begin{tabular}{|c|c|c|c|}
\hline \multirow[b]{2}{*}{ Parámetro } & \multicolumn{2}{|c|}{ CCP } & \multirow[b]{2}{*}{ Valor- P } \\
\hline & $\begin{array}{c}<3.5 \\
(n=13)\end{array}$ & $\begin{array}{c}\geq 3.5 \\
(n=17)\end{array}$ & \\
\hline Aparición del FD (días) & $\begin{array}{l}18.30 \\
\pm 0.99 a\end{array}$ & $\begin{array}{l}15.35 \\
\pm 0.87^{b}\end{array}$ & 0.0340 \\
\hline $\begin{array}{l}\text { Diámetro del FD en la } \\
\text { emergencia }(\mathrm{mm})\end{array}$ & $\begin{array}{l}5.57 \\
\pm 0.08^{\mathrm{a}}\end{array}$ & $\begin{array}{l}5.74 \\
\pm 0.07^{\mathrm{a}}\end{array}$ & 0.1456 \\
\hline Mayor diámetro del FD (mm) & $\begin{array}{l}11.05 \\
\pm 0.13^{\mathrm{b}}\end{array}$ & $\begin{array}{l}11.42 \\
\pm 0.11^{a}\end{array}$ & 0.0466 \\
\hline $\begin{array}{l}\text { Ocurrencia de la ovulación } \\
\text { (días) }\end{array}$ & $\begin{array}{l}32.76 \\
\pm 1.85^{\mathrm{a}}\end{array}$ & $\begin{array}{l}23.94 \\
\pm 1.62^{\mathrm{b}}\end{array}$ & 0.0012 \\
\hline Actividad luteal (días) & $\begin{array}{l}41.38 \\
\pm 2.00^{a}\end{array}$ & $\begin{array}{l}29.64 \\
\pm 1.75^{b}\end{array}$ & 0.0001 \\
\hline $\begin{array}{l}\text { Volumen del cuerpo lúteo } \\
\left(\mathrm{cm}^{3}\right)\end{array}$ & $\begin{array}{l}5.79 \\
\pm 0.49 a\end{array}$ & $\begin{array}{l}7.97 \\
\pm 0.43^{b}\end{array}$ & 0.0025 \\
\hline Progesterona sérica (ng/mL) & $\begin{array}{l}3.16 \\
\pm 0.37^{a}\end{array}$ & $\begin{array}{c}4.18 \\
\pm 0.32^{\mathrm{b}}\end{array}$ & 0.0521 \\
\hline
\end{tabular}

$\mathrm{CCP}=$ Condición Corporal al Parto; ab letras diferentes en los superíndices dentro de cada fila, indican diferencias significativas (t-Student para muestras independientes).
En las vacas con $\mathrm{CCP} \geq 3.5$ el mayor diámetro del FD fue $11.42 \mathrm{~mm}$, superior $(p<0.05)$ que en las de $\mathrm{CC}<3.5$ (Tabla 3). En la totalidad de las hembras con CCP $\geq 3.5$ el FD fue clase III, mientras que en las que tenían $\mathrm{CCP}<3.5$ el $84.6 \%$ fue clase III y $15.4 \%$ fue clase II.

La CCP tuvo una correlación media con la aparición del FD $\left(r=-0.3881, R^{2}=15.06 \%\right.$, $\mathrm{p}=0.0341)$, el mayor diámetro del FD $\left(r=0.366147 ; R^{2}=13.40 \%, p=0.0466\right)$, la ocurrencia de la primera ovulación ( $\mathrm{x}=-0.5605$; $\left.\mathrm{R}^{2}=31.41 \%, \mathrm{p}=0.0013\right)$ y alta con la actividad luteal $\left(r=-0.6399, R^{2}=40.95 \%, p=0.0001\right)$, lo que indica que a mayor CCP, el tiempo en que ocurrieron esos indicadores de la actividad ovárica, expresado días, fue menor. Además, a superior CCP, mayor diámetro del FD y por tanto mayor secreción de $E_{2}$.

Igualmente, la CCP tuvo una correlación media $(p=0.0026)$ con el VCL $\left(r=0.5299, R^{2}=28.08 \%\right)$, $y$ de este con las concentraciones de $\mathrm{P}_{4}$ $\left(r=0.4331, R^{2}=18.76 \%, p=0.0168\right)$; lo que indica que, animales con mejor CCP tienen mayor secresión de $\mathrm{P}_{4}$ y consecuentemente, superior fertilidad posparto.

La CCP inferior a 3.5 puntos, constituyó un factor de riesgo para que las vacas tengan un RAO tardío (Tabla 4).

Tabla 4. Asociación de la CCP con el RAO posparto en las vacas Holstein estudiadas.

\begin{tabular}{lcc}
\hline \multicolumn{1}{c}{ Parámetro } & $\begin{array}{c}\text { Valor } \\
\text { estimado }\end{array}$ & IC 95\% \\
\hline Odds ratio (Probabilidad relativa) & 10.50 & $1.88-58.35$ \\
Fracción atribuible en expuestos & 0.90 & $0.47-0.98$ \\
Fracción atribuible poblacional & 0.67 & $-0.11-0.82$ \\
\hline
\end{tabular}

El Odds ratio, fue altamente significativo $(p=0.0043)$ e indica que los animales con $C C P<3.5$ puntos, tienen 10.50 veces más probabilidades de tener un RAO tardío que los que tienen $\mathrm{CCP} \geq$ de 3.5 puntos.

La Fracción atribuible en expuestos (FAE), denota el porcentaje de las vacas donde el RAO tardío es provocado por tener $\mathrm{CCP}<3.5$ y la Fracción atribuible poblacional (FAP) muestra el porcentaje de ellas dentro de la población total que es causado por la $\mathrm{CCP}<$ de 3.5 puntos $y$, además, la magnitud en que pudiera reducirse el problema si las vacas tuvieran una CCP $a \geq 3.5$ puntos. 


\section{DISCUSIÓN}

El FD en esta investigación apareció más tarde que en lo reportado en vacas Holstein en Colombia, en las que se detectó a los 9.58 \pm 0.6 días en las que tuvieron RAO temprano y a los a los $10.8 \pm 1.50$ días en las que el RAO fue más tardíamente (13). La diferencia puede estar motivada porque en el trabajo consultado los animales recibían suplementación con concentrado y sales minerales.

En este estudio cuando todos los FD alcanzan el mayor tamaño se catalogan como clase III, que son los que tienen diámetro $\geq 10 \mathrm{~mm}$ $(15,16)$; considerando el diámetro de los FD en la emergencia, la tasa de crecimiento folicular fue de un milímetro por día, similar al reportado en vacas Angus y Criollas en Argentina por Robson et al (17).

La pimera ovulación posparto (Tabla 1) con respecto a las vacas Holstein en Colombia, ocurrió más tarde que en las que tuvieron el RAO tempranamente, pero antes que las que la reiniciaron más tardíament, 21.55 y 48.70 días, respectivamente (13).

Aunque en la vaca el RAO posparto ocurre tempranamente y el primer FD se puede detectar entre 10 y 20 días después del parto; los porcentajes de ovulación son bajos y esta ocurre cerca de los 30 días posparto, lo que puede estar condicionado por la pobre concentración y baja secreción pulsátil de LH en este periodo (18).

En el posparto temprano, los folículos se desarrollan y expresan receptores para las hormonas gonadotrópicas (19); sin embargo, el primer FD posterior al parto generalmente no ovula por déficit de LH y cuando lo hace, es tras un celo silente en más del $70 \%$ de las veces y con poca fertilidad a la Inseminación Artificial (15).

Durante las primeras semanas posparto no existen limitaciones del desarrollo folicular provocadas por deficiencia de $\mathrm{FSH}$, pero sí de $\mathrm{LH}$, sobre todo en vacas en BEN. La deficiencia de LH impide el crecimiento, maduración y ovulación del FD de la primera cohorte (20).

Los resultados obtenidos en este estudio indican que el desarrollo del FD hasta tamaño preovulatorio no es limitante en el reinicio de la reproducción posparto de las vacas Holstein, en estas condiciones de producción. Estas hembras tienen un comportamiento reproductivo más favorable que aquellas en las que el RAO posparto se produce después de los 35 DPP, las que tienen menores las tasas de preñez $y$, por tanto, mayores los intervalos parto concepción (21).

Según Adams et al (12), el $46.67 \%$ de las vacas investigadas tuvo ciclos normales, entre 18-24 días y en el $53.33 \%$ los ciclos fueron anormales; en el $6.67 \%$ de ellas fueron $\leq$ que 17 días, que lo clasifican como cortos y el resto son largos, $40 \%$ entre 25 y 30 días y $6.67 \%$ mayor de 30 días.

La duración del ciclo estral entre dos estros consecutivos $\leq$ a 17 días pueden deberse a que el primer FD posparto se desarrolla, pero pocas veces provoca las manifestaciones del estro. No obstante, ocurre la ovulación, se forma el CL y ocurre la luteólisis temprana por la prematura liberación de $\mathrm{PgF}_{2} \mathrm{a}$ endometrial (1). El 40\% de los $\geq$ a 25 días son causa del anestro posservicio, igual que el $6.67 \%$ de los mayores a 30 días, los que también se pueden deber a mortalidad embrionaria precoz (22).

Las diferencias en los parámetros de actividad ovárica posparto (Tabla 3) pueden estar motivadas por los ambios en la CCP, y esta por las concentraciones energéticas de las vacas. Las que tienen $\mathrm{CC}<3.5$ al momento del parto tendrán un BEN más pronunciado y aunque en ellas se produzca la primera ovulación posparto y subsiguientemente la formación del $C L$, este produirá bajos niveles de $\mathrm{P}_{4}(1)$.

El déficit de $\mathrm{P}_{4}$ disminuye la secreción de la $\mathrm{GnRH}$ y la respuesta de la hipófisis a esa hormona; esto provoca ondas atípicas e insuficiente pico preovulatorio de LH que impiden el crecimiento, maduración y ovulación del FD $(20,23)$. Por estas razones las vacas en BEN tienen menores concentraciones circulantes de $\mathrm{P}_{4}$ y Estrógenos $\left(E_{2}\right)(19)$.

Por el contrario, la vaca con $\mathrm{CCP} \geq 3.5$ puntos, tiene reservas energéticas suficientes que garantizan el desarrollo folicular posparto ya que el balance energético positivo influye favorablemente en el ovario y seguidamente el hipotálamo libera $\mathrm{GnRH}$, que estimula la síntesis y liberación de FSH y LH por la hipófisis (19).

En Argentina las vacas mestizas que parieron con CC $>$ de 2.5 puntos tuvieron mayor cantidad de FD clase tres, $\geq 10 \mathrm{~mm}$ de diámetro, mayores porcentajes de estas con CL y concentraciones 
séricas sostenidas de $\mathrm{P}_{4}$ supriores a $0.5 \mathrm{ng} / \mathrm{mL}$ a partir de los 30 DPP. En cambio, en los animales con CC al parto $\leq 2.5$ se retrasóa el RAO (16).

En esta investigación se demostró que la CCP se correlaciona con la actividad ovárica posparto; corroborando los obtenidos por Drescher et al (24), quienes encontraron una correlación $(p=0.0183)$ entre la CCP y la sumatoria de los FD clase tres en ambos ovarios.

En este estuddio se demuestra que la CCP constituye un factor de riesgo significativo para el RAO tardío, corroborando que los estudios de asociación deben ser incorporados al trabajo diario porque ofrecen el impacto del factor de exposición a determinado factor de riesgo (25).

La CCP y BEN posparto pueden afectar el RAO y la fisiología del eje hipotálamo-hipófisis-ovario. Consecuentemente, disminuyen la cantidad de folículos en cada onda folicular, el desarrollo del FD, la frecuencia de liberación de pulsos de LH. Por lo anterior, se retarda la ovulación posparto y disminuye la actividad del $\mathrm{CL}$, especialmente la producción de $\mathrm{P}_{4}(26)$.
Los niveles productivos de los rebaños en esta

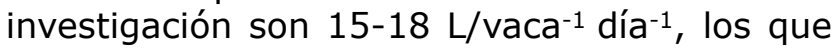
generan requerimientos que no se cubren con una dieta a base de pastos (4) y en estas condiciones se presenta el BEN (27), que se expresa en la CCP, la que influyé sobre el RAO.

Se concluye que el RAO fue temprano, el FD, la ovulación y la actividad luteal ocurren en un periodo de tiempo menor en las vacas con CCP $\geq 3.5$, en las que el $C L$ tiene mayor volumen y son superiores las concentraciones de $\mathrm{P}_{4}$. La CCP constituyó un factor de riesgo para que las vacas tengan el RAO tardío.

\section{Conflicto de intereses}

Los autores de este estudio declaramos que no existe conflicto de intereses con la publicación del presente manuscrito

\section{REFERENCIAS}

1. Henao G. Reactivación ovárica posparto en bovinos. Rev Fac Nal Agr. Medellín. 2001; 54(1):1285-1302. https://revistas. unal.edu.co/index.php/refame/article/ view/24399

2. Ayres H, Ferreira R, Torres-junior J, Demétrio $C$, Sá $M$, Gimenes $L$, et al. Inferences of body energy reserves on conception rate of suckled zebu beef cows subjected to timed artificial insemination followed by natural mating. Theriogenology. 2014; 82(1):529-536. https://doi.org/10.1016/j. theriogenology.2014.04.026

3. Noval E, García-Díaz J.R, García-López R, Quiñones-Ramos R, MollinedaTrujillo A. Caracterización de algunos componentes químicos, en suelos de diferentes agroecosistemas ganaderos. Revista Centro Agrícola. 2014; 41(1):2531. http://cagricola.uclv.edu.cu/index. php/es/?id=79: caracterizacion-de-algunoscomponentes-quimicos-en-suelos-dediferentes-agroecosistemas-ganaderos
4. Balarezo L.R, García-Díaz J.R, HernándezBarreto M.A, García López R. Metabolic and reproductive state of Holstein cattle in the Carchi region, Ecuador Cuban Journal of Agricultural Science. 2016; 50(3):381-392. http://cjascience.com/index.php/CJAS/ article/view/632/699

5. Balarezo LR, García-Díaz JR, Noval-Artíles E, Benavides $H$, Mora S.R, Vargas-Hernández S. Contenido mineral en suelo y pastos en rebaños bovinos lecheros de la región andina de Ecuador. Centro Agrícola. 2017; 44(3):56-64. http://cagricola.uclv.edu.cu/ index.php/es/volumen-44-2017/numero3-2017/939-contenido-mineral-en-suelo-ypastos-en-rebanos-bovinos-lecheros-de-laregion-andina-de-ecuador

6. Balarezo L, García-Díaz J.R, HernánezBarreto M, Vargas-Hernández S. Uterine Involution in Hosltein cows in the province of Carchi, Ecuador. Rev MVZ Córdoba. 2018, 23(2):6649-6659. https://doi. org/10.21897/rmvz.1339 
7. Benavides-Rosales $\mathrm{H}$, Vargas-Hernández $\mathrm{S}$, Aguiar Digna, Rosero D, Pérez L, Rosero M. Assessment of Soil Quality in Andosols Using Silvopastoral Systems. The Open Agriculture Journal. 2018; 12: 207-214. https://doi. org/10.2174/1874331501812010207

8. Rodenburg J. Body Condition Scoring of Dairy Cattle [on line]. OMAFRA Factsheet; 2004. http://www.omafra.gov.on.ca/ english/livestock/dairy/facts/00-109.htm

9. Salas G, Herrera J, Gutiérrez E, Ku-Vera J, Aké-López JR. Reinicio de la actividad ovárica posparto y concentración plasmática de metabolitos lípidos y progesterona en vacas suplementadas con grasa de sobrepaso. Tropical and Subtropical Agroecosystems. 2011; 14(2):385392. http://www.scielo.org. $\mathrm{mx} /$ scielo. php? script $=$ sci arttext\&pid $=S 1870-$ $\underline{04622011000200034 \& \operatorname{lng}=\mathrm{es}}$

10. Walsh R.B, Kelton D.F, Duffield T.F, Leslie K.E, Walton J.S, LeBlanc S.J. Prevalence and risk factors for postpartum anovulatory condition in dairy cows. J Dairy Sci. 2007; 90 (1):315-324. https://doi.org/10.3168/ jds.S0022-0302(07)72632-2

11. Hannan M.A, Fuenzalida M.J, Siddiqui M.A, Shamsuddin M, Beg, M.A, Ginther, O.J. Diurnal variation in $\mathrm{LH}$ and temporal relationships between oscillations in $\mathrm{LH}$ and progesterone during the luteal phase in heifers. Theriogenology. 2010; 74:1491-1498. https://doi.org/10.1016/j. theriogenology.2010.06.021

12. Adams G.P, Jaiswal R, Singh J, Malhi P. Progress in understanding ovarian follicular dynamics in cattle. Theriogenology. 2008; 69(1):72-80. https://doi.org/10.1016/j. theriogenology.2007.09.026

13. Guáqueta $H$, Zambrano J, Jiménez $C$. Factores que afectan la reactivación ovárica posparto en vacas Holstein, en el trópico alto. Rev MVZ Córdoba. 2014; 19(1):39703983. https://doi.org/10.21897/rmvz.117

14. Quintela LA, Barrio M, Peña AI, Becerra JJ, Cainzos J, Herradón PG, et al. Use of ultrasound in the reproductive management of dairy cattle. Reprod Domestic Anim. 2012; 47(Supl 3):34-44. https://doi. org/10.1111/j.1439-0531.2012.02032.x
15. Crowe MA. Resumption of ovarian cyclicity in post-partum beef and dairy cows. Reprod Domestic Anim. 2008. 43(Supl 5):20-28. https://doi.org/10.1111/j.14390531.2008.01210.x

16. Domínguez C, Ruiz A.Z, Pérez R, Martínez $N$, Drescher K., Pinto $L$, et al. Efecto de la condición corporal al parto y del nivel de alimentación sobre la involución uterina, actividad ovárica, preñez y la expresión hipotalámica y ovárica de los receptores de leptina en vaca doble propósito. Rev Fac Cs Vet. 2008; 49(1):23-36. http:// ve.scielo.org/scielo.php?pid $=50258-$ $65762008000100004 \&$ script $=$ sci arttext\&tlng $=\mathrm{es}$

17. Robson C, Aller J.F, Callejas S, Alberio R.H. Dinámica folicular y comportamiento del amamantamiento en razas Angus y criolla Argentina. Arch Zootec. 2008, 57(220):477488. https://www.uco.es/organiza/ servicios/publica/az/php/az.php?idioma gl obal $=0 \&$ revista $=144 \&$ codigo $=1657$

18. Crowe M.A, Diskin, M.G, Williams E.J. Parturition to resumption of ovarian cyclicity: comparative aspects of beef and dairy. Animal. 2014; 8(sup 1):40-53. https://doi. org/10.1017/S1751731114000251

19. Sartori R, Rosa G, Wiltbank M. Ovarian structures and circulating steroids in heifers and lactating cows in summer and lactating and dry cows in winter. J Dairy Sci. 2002; 85(11):2813-2822. https://doi. org/10.3168/jds.S0022-0302(02)74368-3

20. Castro N, Kawashima C, van Dorland HA, Morel I, Miyamoto A, Bruckmaier RM. Metabolic and energy status during the dry period is crucial for the resumption of ovarian activity postpartum in dairy cows. J Dairy Sci. 2012; 95(10):5804-5812. https://doi.org/10.3168/jds.2012-5666

21. Gautam G, Nakao T, Yamada K, Yoshida C. Defining delayed resumption of ovarian activity postpartum and its impact on subsequent reproductive performance in Holstein cows. Theriogenology 2010; 73(2):180-189. https://doi.org/10.1016/j. theriogenology.2009.08.011 
22. Roller Felicia, Pedroso R. Comportamiento Reproductivo, incidencia del anestro y respuesta a rerapéutica hormonal en ganado lechero. Ciencia y Tecnología Ganadera 2007; 1(3):149-159. http://www. actaf.co.cu/revistas/Revista\%20CIMAGT/ Rev.Vol.1\%20No.3,\%202007/Vol\%20 1(3)07Felicia.pdf

23. Maza L, Salgado R, Vergara O. Efecto de la condición corporal al parto sobre el comportamiento reproductivo y variación de peso corporal postparto de vacas mestizas lecheras. Rev MVZ Córdoba. 2001; 6(2):7580. https://doi.org/10.21897/rmvz.526

24. Drescher K, Roa Noris, D'Enjoy D, D'Endel D, Félix-Avellaneda J. Evaluación ultrasonográfica posparto en vacas primíparas Bos Taurus $x$ Bos indicus $\left(F_{1}\right)$ en el trópico. Revista Científica, FCV-LUZ. 2014; 24(4):295-304. http://www.saber. ula.ve/handle/123456789/38949
25. García J.R, Munyori H, Cuesta M, Quiñones R, Figueredo J.M, Noval E, et al. Therapeutic efficacy and pharmacological safety of parenteral supplementation of different concentrations of copper in cows. Archiv Tierzucht. 2012; 55(1):25-35. https://doi. org/10.5194/aab-55-25-2012

26. Santos J.E, Rutigliano H.M, Sa Filho M.F. Risk factors for resumption of postpartum estrous cycles and embryonic survival in lactating dairy cows. Animal Reproduction Science. 2009; 110(3-4):207-221. https:// doi.org/10.1016/j.anireprosci.2008.01.014

27. Scheneider J. Energy balance and reproduction. Physiol Behav. 2004; 81 (2):289-317. https://doi.org/10.1016/j. physbeh.2004.02.007 